\title{
Analysis of Decision Making Operation in Cognitive radio using Fuzzy Logic System
}

\author{
Maninder Jeet Kaur \\ Department of Computer Science and \\ Engineering \\ National Institute of Technology, \\ Jalndhar, Punjab, India
}

\author{
Moin Uddin \\ Director, \\ National Institute of Technology, \\ Jalndhar, Punjab, India
}

\author{
Harsh K. Verma \\ Department of Computer Science and \\ Engineering \\ National Institute of Technology, \\ Jalndhar, Punjab, India
}

\begin{abstract}
Introduction of flexibility and intelligence in the wireless devices and applications have introduced the concept of Cognitive Radio. This research objective has inspired various research activities on going which included the decision making aspects. In this paper, a decision making process in cognitive radio is analyzed using fuzzy logic system, in which secondary user can use the spectrum effectively. We have selected three descriptive factors for choosing the proper secondary unlicensed user - velocity of the secondary user, spectrum to be utilized by secondary user and distance of the secondary user from primary user. The efficiency of the decision making process in cognitive radios is analyzed. Based on linguistic knowledge 27 rules are set up. The output of the fuzzy logic system gives the probability of the decision based on the three descriptive factors. We show how fuzzy logic system can be used for decision making operation in cognitive radio.
\end{abstract}

\section{Keywords}

Cognitive Radio, Fuzzy Logic

\section{INTRODUCTION}

In past few years researchers have studied how the wireless devices can be used in dynamic and partially known envoirnments. This has lead to new research field named Cognitive Radio, a term introduced by Joseph Mitola[1]. Main functions of the Cognitive Radio are spectrum sensing, spectrum mobility, spectrum sharing and optimization of the resources. With these functions a model is proposed where an unlicensed user can utilize available licensed spectrum in dynamic manner depending on the possibility of access based on external parameters. Different researches are in progress and ways are being found to efficiently utilize the available licensed spectrum. One of the ways is the use of "Cognitive Radio", according to this; the already licensed spectrum can be used more efficiently by introducing artificial intelligence, the decision- making to be specific, in the radio. This enables the radio to learn from its environment, considering certain parameters. Based on this knowledge the radio can actively exploit the possible empty frequencies in the licensed band of the spectrum that can then be assigned to other processes in such a way that they don't cause any interference to the frequency band that is already in use. This makes the efficient usage of the available licensed spectrum possible.

Fuzzy Logic was initiated in 1965 [2, 3, 4] by Lotfi A. Zadeh , professor for computer science at the University of California in Berkeley. Basically, Fuzzy Logic (FL) is a multivalued logic that allows intermediate values to be defined between conventional evaluations like true/false, yes/no, high/low, etc. We note that Fuzzy Logic has been already proposed for use in the context of telecommunication system, e.g., for QoS routing in wired networks [5], route caching decisions in wireless adhoc networks [6], radio resource management [7] and channel selection in cellular networks [8]. An interesting survey on the usage of Fuzzy Logic techniques in the telecommunication field can be found in [9]. In fuzzy logic system, knowledge of a restricted domain is captured in the form of linguistic rules. Relationships between two goals are defined using fuzzy inclusion and non-inclusion between the supporting and hindering sets of the corresponding goals [10]. Fuzzy Logic has been successfully applied in various areas pertaining to wireless communication systems. As fuzzy logic is used to model systems and situations, taking into consideration uncertainty and ambiguity, it can be an efficient tool to be utilized in problems for which knowledge of all factors is insufficient or impossible to obtain.

The contents of the paper are as follows: Section II gives as overview of Cognitive Radio Technology; Section III describes some concepts about Fuzzy Logic System, Section IV we show the proposed model of our study. Section V gives the simulation results. Section VI presents the conclusions.

\section{COGNITIVE RADIO TECHNOLOGY}

A cognitive radio is a new technology that can intelligently adapt its behavior or operational characteristics in response to changes in the radio system's internal state or external environment. This ability to adapt promises a range of functional capabilities, including making the radio system more personal as conceptualized by Mitola [11]. The conservation of the spectrum is a priority in international regulatory planning since then. FCC found the spectrum utility of $55-95 \%$ below a spectrum of $1 \mathrm{GHz}$ [12]. SSC (Shared Spectrum Company) reported the maximum occupancy of $13.1 \%$ [13].

Key Concepts in Cognitive Radio:

- $\quad$ Primary Network

○ An existing network architecture which has as access right to certain spectrum band

$\circ$ e.g. Common Cellular systems and TV broadcast networks

- $\quad$ Primary User(Licensed User)

- Has a license to operate in a certain spectrum band 
- $\quad$ Secondary Network(Unlicensed Network)

- Does not have license to operate in a desired band

- Hence, the spectrum access is allowed in an opportunistic manner

- Secondary User(Unlicensed User)

○ Has no spectrum license

- Hence, additional functionalities are required to share the licensed spectrum band

By sensing and adapting to the environment a cognitive radio is able to fill the spectrum holes and serve's its users without causing harmful interference to the licensed users [14].

Cognitive radio uses the spectrum effectively by the four main steps [15]:

1. Spectrum Sensing: It refers to detect the unused spectrum and sharing it without harmful interference with other users. It is an important requirement of the Cognitive Radio network to sense spectrum holes, detecting primary users is the most efficient way to detect spectrum holes.

2. Spectrum Management: It is the task of capturing the best available spectrum to meet user communication requirements.

3. Spectrum Mobility: It is defined as the process where the cognitive user exchanges its frequency of operation

4. Spectrum Sharing: This refers to providing a fair spectrum scheduling method among the users. Sharing is the major challenge in the open spectrum usage.

Cognitive Radio adopts the Opportunistic Spectrum Sharing approach [16], which allows the SUs to coexist with Primary Users (PUs) who are the legacy spectrum holders. PUs has provision to access communication channels at any time [17]. SUs need to sense the radio environment, and opportunistically utilize available spectrum. Meanwhile, they need to adapt to local behaviors of PUs and vacate the spectrum immediately when the presence of primary users is detected [18].

The design of cognitive radio is very challenging due to complexity, modularity, information imprecision and interpretability issues. Fuzzy logic system is chosen for the decision making because it is very much suited for non linear, imprecision and multivalued problems as it is capable of making real time decisions.

\section{FUZZY LOGIC}

A very brief overview of fuzzy logic is provided here, mentioning the concepts of fuzzy sets, fuzzy logic inference and fuzzy control system. The purpose of this overview is to help reader get familiar with fuzzy logic.

Fuzzy Logic is convenient way to map input space to output space. It is a multivalued logic that allows intermediate values to be defined between conventional evaluations like true/false, yes/no, high/low etc.
Fuzzy Sets - In fuzzy logic, each object x can be labeled by a linguistic term, where a linguistic term is a word such as "small", "medium", "large", etc so that $x$ is defined as a linguistic variable. Each linguistic variable is associated with a term $F(x)$, which is the set of names of linguistic values of $x$. Each element in $F(x)$ is a fuzzy set.

Membership functions - The membership function is a graphical representation of the magnitude of participation of each input. It associates a weighting with each of the inputs that are processed, define functional overlap between inputs, and ultimately determines an output response. The rules use the input membership values as weighting factors to determine their influence on the fuzzy output sets of the final output conclusion.

If $\mathrm{X}$ is a collection of objects denoted generally by $x$, then a fuzzy set $\mathrm{A}$ in $X$ is defined as a set of ordered pairs:

$\mathrm{A}=\left\{\left(x, \mu_{A}(x)\right) x \in X\right\}$,

where $\mu_{\mathrm{A}}$ is called the membership function (MF) for the fuzzy set A. The MF maps each element of $X$ to a membership grade (membership value) between 0 and 1 . Usually $X$ is referred to as the universe of discourse, or simply the universe and it may consist of discrete (ordered or nonordered) objects or continuous space [19]. To implement a decision making processes, fuzzy logic makes use of the so called Fuzzy Logic Controllers (FLCs). The essential part of the FLC is a set of linguistic control rules based on expert knowledge in the form:

IF (a set of conditions are satisfied) THEN (a set of consequences can be inferred)

Linguistic rules describing the control system consist of two part; an antecedent block (between the IF and THEN) and a consequent block (following THEN). Depending on the system, it may not be necessary to evaluate every possible input combination, since some may rarely or never occur. The inputs are combined logically using the AND operator to produce output response values for all expected inputs. The active conclusions are then combined into a logical sum for each membership function. A firing strength for each output membership function is computed. All that remains is to combine these logical sums in a defuzzification process to produce the crisp output.

A general FLC consists of four modules: a fuzzy rule base, a fuzzy inference engine and a fuzzification /defuzzification module. A FLC operates by repeating a cycle of five steps implemented by these four modules. First, measurements are taken of all variables that represent relevant conditions of the controlled process. Next, these measurements are converted into appropriate fuzzy sets to express measurement uncertainties. This step is called fuzzification. The fuzzified measurements are then used by the inference engine to evaluate control rules stored in the fuzzy rule base. The reasoning (Inference) is to select the most appropriate rule at a certain condition. The result of this evaluation is a fuzzy set (or several fuzzy sets) defined on the universe of discourse of possible actions. This fuzzy set is the converted, in the final step of the cycle, into a crisp value (or a vector of values). This conversion is called defuzzification. The defuzzified values represent actions taken by the FLC in individual control cycles $[20,21]$. 
The fuzzy outputs for all rules are finally aggregated to one fuzzy set. To obtain a crisp decision from this fuzzy output, we have to defuzzify the fuzzy set, or the set of singletons. Therefore we have to choose one representative value as the final output. Rules can be solved in parallel in hardware, or sequentially in software. The results of all the rules that have fired are "defuzzified" to a crisp value by one of of several methods. There are dozens in theory, each with various advantages and drawbacks. The "centroid" method is very popular, in which the "center of mass" of the result provides the crisp value. Table I summarizes the questions used in the survey. Since we use a single consequent for each rule to form a rule base, we averaged the centroids of all the responses for each rule and used this average in place of the rule consequent centroid. Doing this leads to rules that have the following form.

IF spectrum utilization efficiency of the secondary user $\left(x_{l}\right)$ is $F_{l}^{l}$, and velocity $\left(x_{2}\right)$ is $F_{l}^{2}$ and its distance $\left(x_{3}\right)$ to the primary user is $F_{l}^{3}$, THEN the possibility $(y)$ that this secondary user is chosen to access the available spectrum is $c^{l}{ }_{\text {avg }}$

Where $1=1,2, \ldots \ldots .27$ and $\mathrm{c}^{1}{ }_{\text {avg }}$ is defined as follows:

$$
c_{\text {avg }}^{l}=\frac{\sum_{i=1}^{5} w_{i}^{l} c^{i}}{\sum_{i=1}^{5} w_{i}^{l}}
$$

in which $w_{i}^{l}$ is the number of experts choosing linguistic label I for the consequence of rule $l$ and $c^{i}$ is the centroid of the $i$ th consequence $\operatorname{set}(1=1,2, \ldots .5 ; 1=1,2, \ldots \ldots .27)$.

For every input $\left(x_{1}, x_{2}, x_{3}\right)$, the output $y\left(x_{1}, x_{2}, x_{3}\right)$ of the designed system is computed as

$$
y\left(x_{1}, x_{2}, x_{3}\right)=\frac{\sum_{l=1}^{27} \mu_{F_{1}}\left(x_{1}\right) \mu_{F_{2}^{l}}\left(x_{2}\right) \mu_{F_{3}^{l}}\left(x_{3}\right) c_{a v g}^{1}}{\sum_{i=1}^{27} \mu_{F_{l}^{1}}\left(x_{1}\right) \mu_{F_{2}^{1}}\left(x_{2}\right) \mu_{F_{3}^{l}}\left(x_{3}\right)}
$$

We recognize that equation above can be represented in a 4D surface. Since it is impossible to plot visually, we fix one of the three factors.

\section{PROPOSED MODEL}

In the proposed model of the study for selecting the best suitable secondary user to access the spectrum, the determining parameters which are considered are: velocity of the secondary user, spectrum to be utilized by the secondary user and distance of the secondary user from the primary user. Velocity (Mobility) is an important parameter because more will be the chances of secondary user to change the position. It will cause Doppler Effect.

$$
f_{D}=\frac{v \cos \theta}{c} f_{c}
$$

$f_{D}$ is the Doppler shift, $\theta$ is the arrival angle of the received signal, $c$ is the wave velocity and $f c$ is the carrier frequency. If the velocity is more there can be degradation of Quality of service due to non availability of the channel. The velocity can be calculated from the given formula. Doppler shift is given by,

$$
f=f_{m} \cos \varphi
$$

where $f_{m}=\frac{v}{\lambda}$,

$f_{m}$ is the carrier frequency,

$v$ is the mobile velocity,

$\varphi$ is the angle,

$\lambda$ is the carrier wave length

Distance of the secondary user is another important factor because secondary user at a closer distance should be given priority to access spectrum, which depends upon the SNR, $\gamma_{s}$, at the secondary user given by,

$$
\gamma_{s}=10 \log \left(\left(P_{1} g(R)\right) / \sigma^{2}\right)
$$

where $\mathrm{P}_{1}$ is the transmit power user, $\sigma$ is the noise power and $\mathrm{R}$ is the distance between the primary and the secondary user which can be calculated from the given formula. PHY Layer could exploit knowledge of modulation and coding schemes being used to translate SNR measurements in $\mathrm{dB}$ into more abstract fuzzy attributes bad or good. The result of this fuzzification is that the fuzzy SNR good or bad characterization can be correctly interpreted even without explicit knowledge regarding modulation and coding, since we can rely on the fact that they have been taken into account for the fuzzification process.

The third factor is the ratio of the required spectrum to the available spectrum which gives spectrum efficiency given by,

$$
\eta_{s}=\left(B W_{S} / B W_{a}\right) * 100 \%
$$

where $\mathrm{BW}_{\mathrm{s}}$ is the required spectrum and $\mathrm{BW}_{\mathrm{a}}$ is the available spectrum. Table 1 shows the rules corresponding to the parameters used for decision making operation.

This research paper focuses on the spectrum management and mobility issues in the cognitive radio with an assumption that the sensing information is provided as an input either from the radio environment or the secondary user itself specifies the QoS requirements specifications to the radio.

The cognitive radio receives the RF environment at its receiver and involves itself in the decision making process to accommodate the new user requesting the spectrum allocation.

Table 1: Questions For Each Rule Corresponding To The Problem In Cognitive Radio

\begin{tabular}{|l|l|l|l|l|}
\hline $\begin{array}{l}\text { Rule } \\
\text { No. }\end{array}$ & Spectrum & Velocity & Distance & Decision \\
\hline 1 & Low & Low & Near & Very Low \\
\hline 2 & Low & Low & Moderate & Low \\
\hline 3 & Low & Low & Far & Low \\
\hline 4 & Low & Moderate & Near & Very Low \\
\hline 5 & Low & Moderate & Moderate & Low \\
\hline 6 & Low & Moderate & Far & Moderate \\
\hline 7 & Low & High & Near & Very Low \\
\hline
\end{tabular}




\begin{tabular}{|l|l|l|l|l|}
\hline 8 & Low & High & Moderate & Low \\
\hline 9 & Low & High & Far & Moderate \\
\hline 10 & Medium & Low & Near & Very Low \\
\hline 11 & Medium & Low & Moderate & Moderate \\
\hline 12 & Medium & Low & Far & High \\
\hline 13 & Medium & Moderate & Near & Very Low \\
\hline 14 & Medium & Moderate & Moderate & Moderate \\
\hline 15 & Medium & Moderate & Far & High \\
\hline 16 & Medium & High & Near & Very Low \\
\hline 17 & Medium & High & Moderate & Low \\
\hline 18 & Medium & High & Far & High \\
\hline 19 & High & Low & Near & Low \\
\hline 20 & High & Low & Moderate & High \\
\hline 21 & High & Low & Far & Very High \\
\hline 22 & High & Moderate & Near & Low \\
\hline 23 & High & Moderate & Moderate & High \\
\hline 24 & High & Moderate & Far & Very High \\
\hline 25 & High & High & Near & Very low \\
\hline 26 & High & High & Moderate & High \\
\hline 27 & High & High & Far & High \\
\hline
\end{tabular}

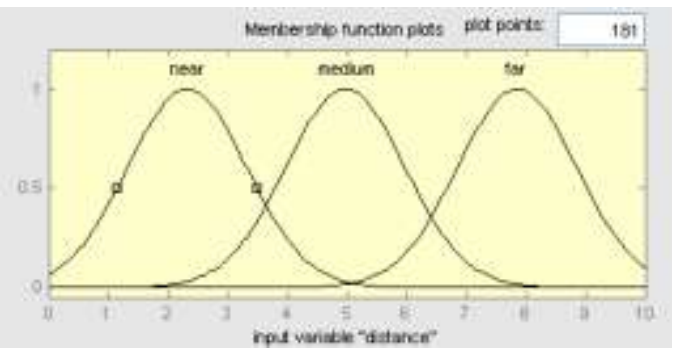

Fig 3: Membership Function Plot for Distance

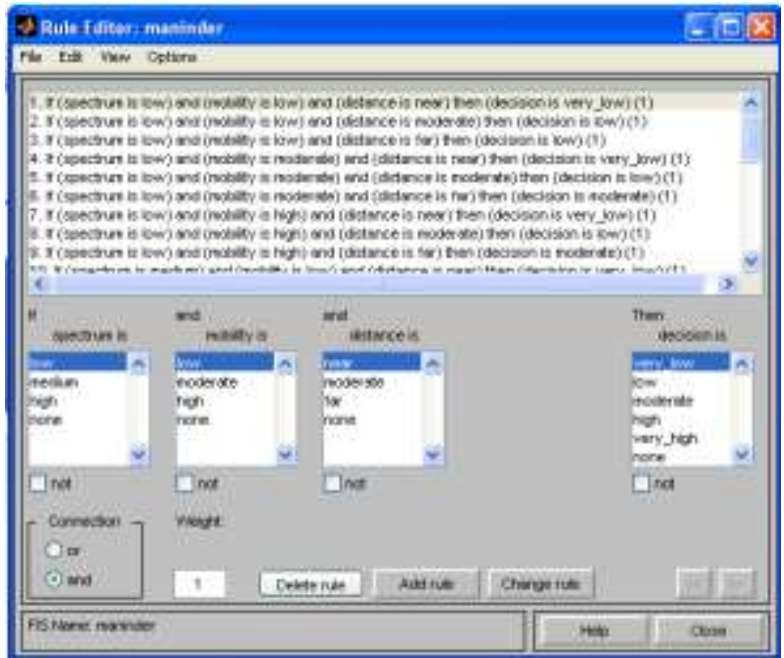

Fig 4: Rule Editor 7.9. The three descriptors are used as the inputs to the system and the opportunistic spectrum access decision possibility is the output of the system.

The descriptive linguistic variables of velocity and the spectrum to be utilized by the secondary user are divided into three levels i.e. low, medium and high, while the distance is divided into near, medium and far

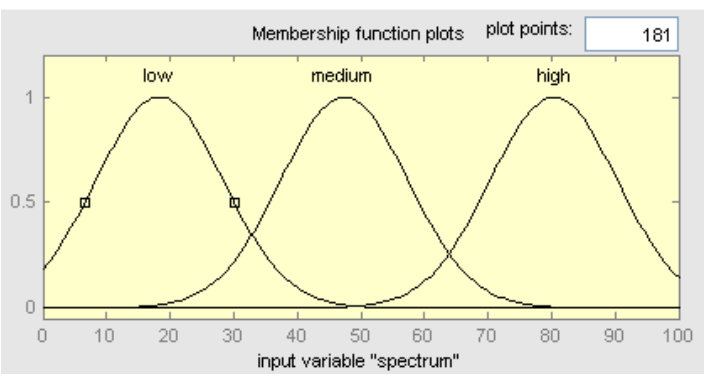

Fig 1: Membership Function Plot for Spectrum

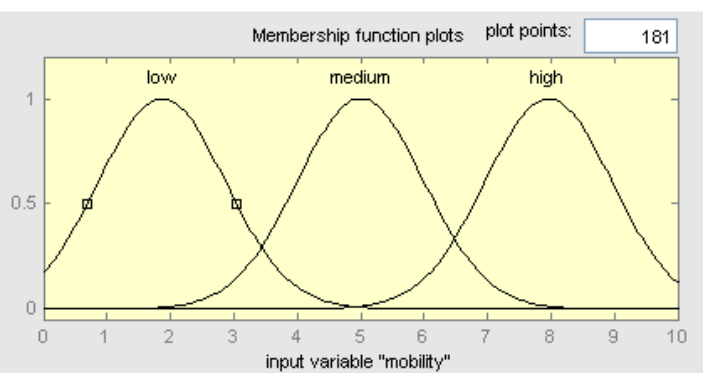

Fig 2: Membership Function Plot for Velocity

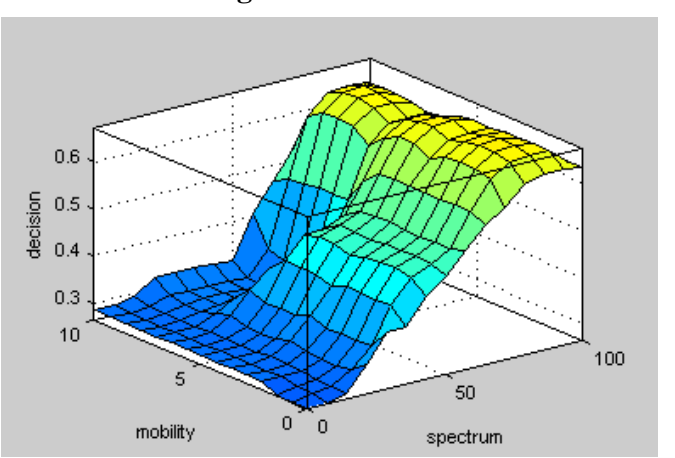

Fig 5: Decision surface showing mobility and spectrum

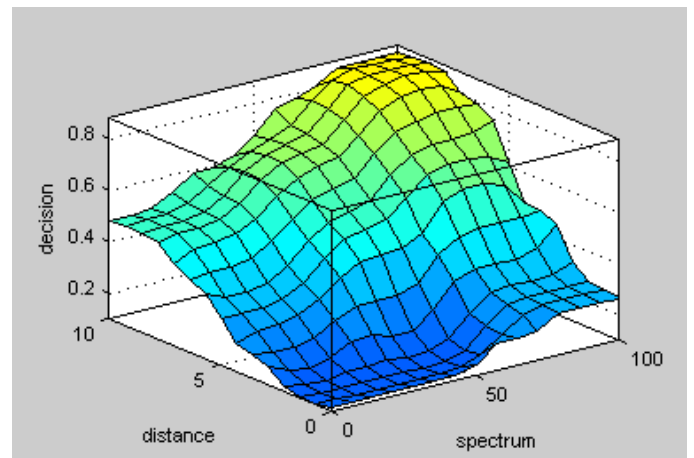

Fig 6: Decision Surface showing distance and spectrum 


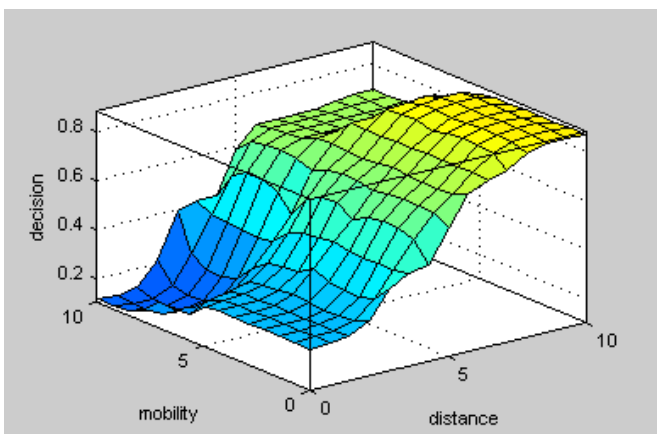

Fig 7: Decision Surface showing mobility and distance

From the simulation results, it shows that the chance of decision is affected by the distance of the secondary user and the primary user. The less the distance, the more the probability of the decision. It also depends on the velocity of the secondary user. The more the velocity, the more is the probability for the secondary user to have access to the spectrum.

\section{CONCLUSION}

Fuzzy Logic provides a different way to approach a control or classification problem. Fuzzy approach requires a sufficient expert knowledge for the formulation of the rule base, the combination of the sets and the defuzzification. The employment of fuzzy logic might be helpful, for very complex processes, when there is no mathematical model for highly non-linear processes or if the processing of expert knowledge is to be performed. The results show that the chance of decision increases if the distance between licensed and unlicensed user is low and velocity of the secondary user is more. The decision making operation will enable the cognitive radio access the spectrum opportunistically which will in turn improve spectrum utilization. It is important to investigate some solutions to prevent some users from using spectrum ineffectively and solve the mobility management problem in order to keep a high QoS of Cognitive Radio.

\section{REFERENCES}

[1] J. Mitola and G.Q. Maguire. 1999. Cognitive radio: making software radios more personal. Personal Communications. IEEE, 6:13-18.

[2] L. A. Zadeh. 1965. Fuzzy Sets. Information and Control.

[3] L. A. Zadeh. 1973. Outline of A New Approach to the Analysis of Complex Systems and Decision Processes.

[4] L. A. Zadeh. 1968. Fuzzy algorithms. Information and Control., Vol. 12, pp. 94-102.

[5] R. Zhang and K. Long. 2002. A fuzzy routing mechanism in next generation networks. In IASTED International Conference on Intelligent Systems and Control, Tsukuba City, Japan.

[6] S. Rea and D. Pesch. 2004. Multi-metric routing decisions for ad hoc networks using fuzzy logic. In International Symposium on Wireless Communication Systems, Mauritius.
[7] L. Giupponi, R. Agusti, J. P'erez-Romero, and O. Sallent. 2005. Joint radio resource management algorithm for multiRAT networks. In IEEE Globecom, St. Louis, USA.

[8] M. Abdul-Haleem, K. Cheung, and J. Chuang. 1995. Aggressive fuzzy distributed dynamic channel assignment algorithm. In Proc. IEEE International Conference on Communications, Vol. 1.

[9] S. Ghosh, Q. Razouqi, H. Schumacher, and A. Celmins. 1998. A survey of recent advances in fuzzy logic in telecommunications networks and new challenges. IEEE Transactions Fuzzy Systems, Vol. 6, No. 3, pp. 443- 447.

[10] R. Fouler, C . Carlsson. 1996. Fuzzy multiple criteria decision making: Recent developments. In Fuzzy Sets and Systems, Vol. 78, pp 139-153.

[11] J. Mitola. 2001. Cognitive Radio for Flexible Mobile Multimedia Communications. Springer Mobile Networks and Applications, pp 435-441, Vol 6, No. 5.

[12] Federal Communication Commission (FCC). 2004. Unlicensed operation in TV Broadcast Bands. Notice for proposed Rule Making, ET Docket No. 04-113, May 25.

[13] Shared Spectrum Company. 2005. Spectrum Occupancy Measurements. http://www.sharedspectrum .com/measurements/

[14] C. Sun, W. Zhang and K. Ben Lataief. 2007. Cluster-based cooperative spectrum sensing in cognitive radio systems. In Proc IEEE International Conference Communication., Glasgow, Scotland, UK, June 24-28, pp. 2511-2515.

[15] Ian F, Akyildiz, Won-Yeol Lee, Mehmet C. Vuran, Shantidev Mohanty. 2006. NeXt Generation/Dynamic Spectrum Access/ Cognitive Wireless Networks: A Survey. Computer Networks, pp 2127- 2159.

[16] T. Weiss and F. Jondral. 2004. Spectrum pooling: An innovative strategy for the enhancement of spectrum efficiency. IEEE Communication Magazine, Vol.42, pp. S8S14.

[17] S. Haykin. 2005. Cognitive radio: Brain-empowered wireless communications. IEEE Journal on Selected Area Communication, Vol. 23, No. 2, pp. 201-220.

[18] S. Mangold, S. Shankar, and L. Berlemann. 2005. Spectrum agile radio: A society of machines with value-orientation. In Proceedings of 11th European Wireless Conference, vol. 2, pp. 539-546, Nicosia, Cyprus.

[19] J.S.R. Jang, C.T. Sun and E.Mizutani. Neuro-Fuzzy and Soft Computing A computational Approach to Learning and Machine Intelligence.

[20] A. Kaufmann. 1975. Introduction to Theory of Fuzzy Subsets, New York: Academic.

[21] H.J. Zimmermanm. 1987. Fuzzy Sets, Decision Making and Expert Systems, Boston: Kluwer Academic Publisher. 